\title{
Percutaneous Cementoplasty for the Pelvis in Bone Metastasis: 12-Year Experience
}

\author{
Jong Woong Park, MD ${ }^{1,2}$, Hyun-ju Lim, MD, $\mathrm{PhD}^{3}$, Hyun Guy Kang, MD, PhD ${ }^{1,2}$, June Hyuk Kim, MD ${ }^{1}$, \\ and Han-Soo Kim, MD, PhD $^{4}$ \\ ${ }^{1}$ Orthopaedic Oncology Clinic, National Cancer Center, Goyang, Gyeonggi-do, Republic of Korea; ${ }^{2}$ Division of \\ Convergence Technology, National Cancer Center, Goyang, Gyeonggi-do, Republic of Korea; ${ }^{3}$ Department of Radiology, \\ National Cancer Center, Goyang, Gyeonggi-do, Republic of Korea; ${ }^{4}$ Department of Orthopaedic Surgery, Seoul National \\ University Hospital, Seoul, Republic of Korea
}

\begin{abstract}
Background. In advanced cancer patients, pelvic bone metastasis often causes pain and gait disturbance. The use of percutaneous bone cement [polymethylmethacrylate (PMMA)] injection for pain management and strengthening in pelvic bone metastasis has rarely been reported. To evaluate this method, we aimed to determine surgical outcomes and complications over a long-term follow-up period using a large patient group.

Patients and Methods. We retrospectively collected data from 178 patients who underwent percutaneous cementoplasty for pelvic metastatic lesions, 201 in total. Surgical outcomes evaluated included pain reduction and improvement of ambulation. Mortality within 1 month after procedure and pulmonary embolism caused by thrombus, fat, tumor emboli, or bone cement were investigated as surgical complications. For long-term survivors, pain relapse and mechanical failure were analyzed. The mean follow-up period was 12.6 months, and there were 159 fatalities at last follow-up.

Results. The mean regional pain numerical rating scale scores decreased from 6.1 preoperatively to 2.41 month after procedure $(p<0.01)$. Gait function was maintained, worsened, and uncheckable in $68 \%, 24 \%$, and $8 \%$ of patients, respectively, 1 month after procedure. Of long-
\end{abstract}

(C) Society of Surgical Oncology 2021

First Received: 8 March 2021

Accepted: 24 July 2021;

Published Online: 26 August 2021

H. G. Kang, MD, PhD

e-mail: ostumor@ncc.re.kr term survivors followed up for $>12$ months $(n=53)$, there were no significant changes in serial plain radiographs, and regional pain aggravation was observed in 9\%. Pulmonary cement embolism and bone cement implantation syndrome was observed in $11 \%$ and $10 \%$, respectively. However, all patients with these complications were asymptomatic.

Conclusions. Percutaneous cement injection into the pelvis is a feasible and safe palliative surgical option for patients with advanced malignancy in terms of pain reduction and maintenance of ambulatory function under regional anesthesia.

The effectiveness of surgery for bone metastasis in advanced cancer patients is limited, but it can greatly improve patients' quality of life. In most patients with advanced-stage bone metastatic cancer, complete resection or extended curettage of the bone metastasis with major reconstruction is often not possible owing to their poor general condition and other accompanying metastases. This is especially true for pelvic metastatic lesions because the surgery is particularly burdensome. ${ }^{1-4}$ Pain relief and maintenance of gait function are therefore the main goals of palliative treatment in these patients. ${ }^{1-5}$

Percutaneous bone cement injection with polymethylmethacrylate (PMMA) has been used for pain management and strengthening of various anatomical sites. While it is often used for vertebroplasty or kyphoplasty in the spine, it can also be useful in augmenting the stability of regions other than the spine ${ }^{6-10}$ either alone, or in conjunction with metallic fixtures. It can also be successfully used in metastatic bone lesions, where it provides mechanical stability to the damaged bone and exerts a hyperthermal 
effect on tumor cells. ${ }^{11,12}$ For pelvic metastasis, bone cement injection without metallic fixation is performed as a palliative minimal invasive surgery, and the resultant surgical outcomes have been reported in several studies. ${ }^{13-20}$ However, many studies were small case series or excluded advanced cancer patients with a short life expectancy for whom a minimal invasive procedure was theoretically most beneficial. Moreover, pulmonary cement embolism after PMMA injection needed to be addressed.

This study aimed to summarize the surgical outcomes of percutaneous cementoplasty for pelvic metastases using data from a large group of advanced cancer patients to investigate the effectiveness and complications of the procedure.

\section{PATIENTS AND METHODS}

\section{Patients}

Using the institutional database, data were retrospectively collected from 178 consecutive patients, with a total of 201 pelvic bones, who underwent percutaneous cementoplasty for pelvic metastatic lesions from January 2008 to December 2019 (Fig. 1). The primary outcome of this study was surgical outcomes in terms of pain reduction and maintenance of gait function, and secondary outcomes were complications of the procedure. This study was approved by the National Cancer Center Institutional Review Board. The requirement for written informed consent from the patients was waived due to the retrospective study design.

The indication for the procedure was advanced cancer patients (1) who had refractory pain and gait or disturbance, (2) with osteolytic lesion of the pelvis in the area suitable for the pain, and (3) with short life expectancy or stopping active medical treatment. In patients with painful osteolytic lesion in the pelvis who had not previously undergone radiotherapy, treatment was often performed first, followed by radiotherapy. There was no absolute contraindication of the procedure; however, the relative contraindication was advanced cancer patients (1) with stable course or (2) with long life expectancy (more than a few years). These patients were often considered candidates for major pelvic surgery including tumor removal and hip arthroplasty. If patients' general condition allowed minimal anesthesia, short life expectancy or poor general condition was not considered a contraindication. In order of decreasing frequency, primary diagnoses were lung cancer
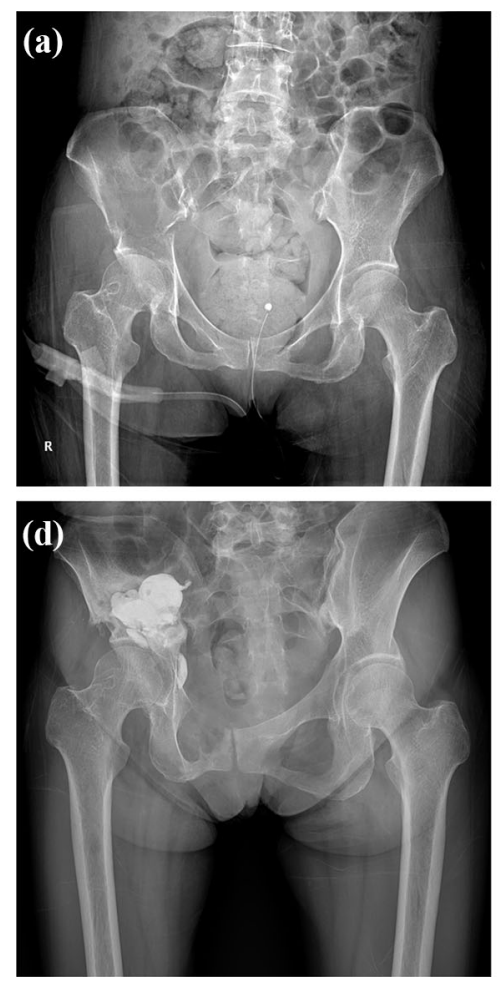

FIG. 1 Representative images for percutaneous pelvic cementoplasty. a A plain radiograph, b computed tomography image, and c T1-enhanced magnetic resonance image showing osteolytic bone metastasis with a cortical defect on the right acetabulum. d A postoperative plain radiograph showing reinforced

(e)
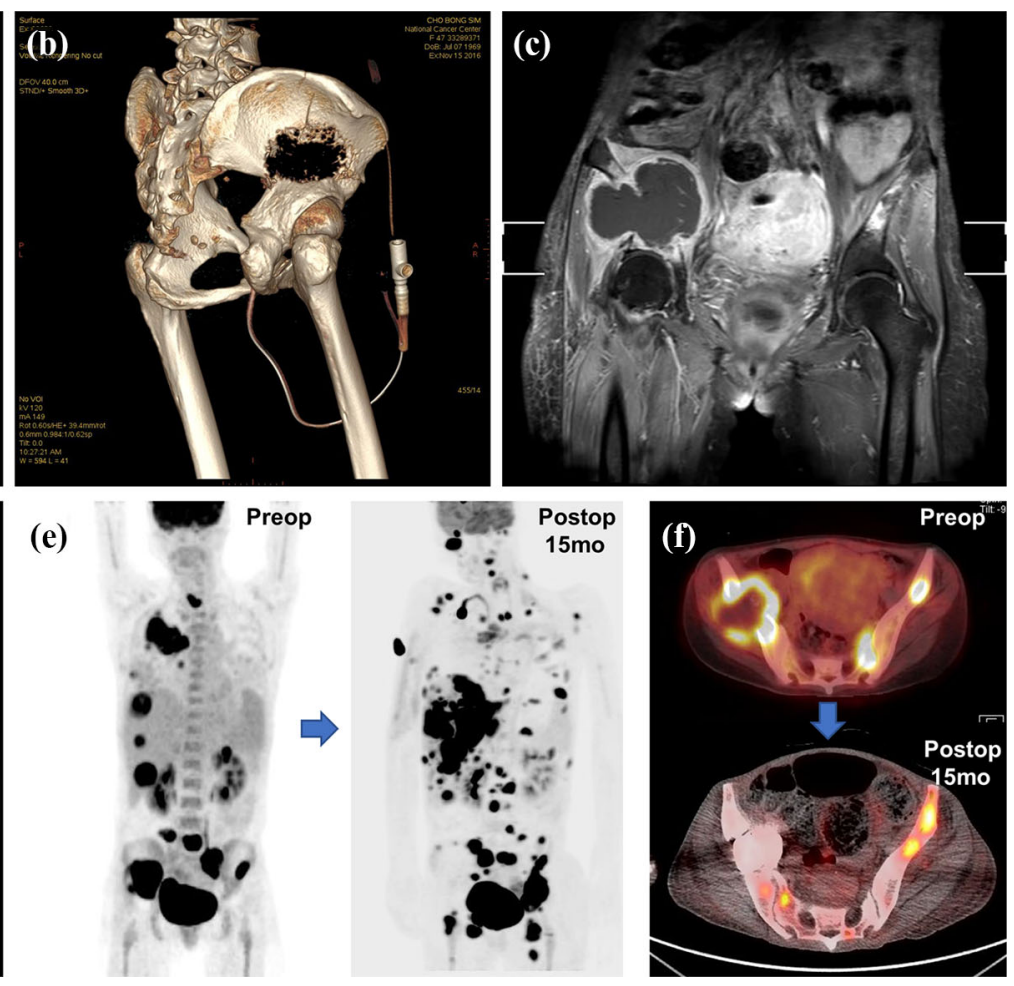

acetabulum. e, f Positron emission tomography-computed tomography (PET-CT) images at the time of preoperative (e, left; $\mathbf{f}$, upper) and 15-month postoperative status (E, right; $F$, lower) showing disease progression except at the right acetabulum 
( $n=58)$, breast cancer $(n=45)$, hepatocellular carcinoma (HCC) $(n=13)$, renal cell carcinoma (RCC) $(n=12)$, prostate cancer $(n=11)$, colorectal cancer $(n=10)$, thyroid cancer $(n=7)$, bladder/ureter cancer (transitional cell cancer) $(n=5)$, sarcoma $(n=5)$, and others $(n=12)$. No patients had solitary or oligometastasis (less than three sites). Of all the patients included in the study, 96 underwent percutaneous cementoplasty for the pelvis alone and 82 underwent other minimally invasive procedures concurrently with the procedure (femur: $n=71$, femur and tibia: $n=4$, humerus: $n=4$, tibia: $n=1$, vertebroplasty: $n=$ 1, and sternum: $n=1)$. Among the 96 patients who underwent only percutaneous cementoplasty, 88 underwent single-side procedure and 8 underwent procedure for both sides. The mean times for the procedure were $28 \mathrm{~min}$ (range 11- $67 \mathrm{~min}$ ) and $51 \mathrm{~min}$ (range 37-82 $\mathrm{min}$ ) for single and both sides of pelvis, respectively. Regional spinal anesthesia was administered for most operations $(n=$ 140). The 38 patients who underwent surgery under general anesthesia concurrently underwent surgery for other areas of the body. The mean quantity of bone cement used for single-side pelvis was $21.8 \mathrm{ml}$ (range $7-50 \mathrm{ml}$ ). All patients who had no contraindication for deep vein thrombosis prophylaxis were administered an anticoagulant (enoxaparin 40-60 mg per day) from 1 day before the procedure until ambulation was resumed $(n=119,67 \%)$. The mean follow-up period was 12.6 months, and there were 159 fatalities by last follow-up (Table 1).

The extent of the periacetabular defect was evaluated using preoperative, pre-enhanced T1-weighted magnetic resonance imaging (MRI). The metastatic pelvic lesions treated by percutaneous cementoplasty commonly involved the acetabular dome (185/201 pelvic lesions, 92\%). For the 16 lesions without acetabular dome involvement, 10 were iliac lesions near the sacroiliac joint, 5 were ischial lesions in contact with the medial wall of the acetabulum, and 1 was a superior pubic ramus lesion. Among the 185 lesions with acetabular dome involvement, 78 involved the acetabular dome alone, 50 extended to the posterior column, 10 extended to the anterior column, and 47 extended to both columns.

Surgical efficacy was evaluated by pain reduction and improvement of ambulation. Evaluation of pain and gait function was conducted preoperatively, and at 1 week and 1 month postoperatively. To evaluate pain, numerical pain score (NRS) was used, and tolerable pain was defined as mild pain with 4 points as cut-off. ${ }^{21}$ For long-term survivors who were followed up for more than 1 year, pain relapse or mechanical failure was analyzed.

Surgical complications included mortality within 1 month after procedure, pulmonary embolism, and bone cement implantation syndrome (BCIS). Bone cement implantation syndrome was defined as "hypoxia,
TABLE 1 Demographics

\begin{tabular}{ll}
\hline Characteristics & No. (\%) \\
\hline Age at procedure (years) & 60.5 (range 23-87) \\
Sex & \\
Male & $80(45)$ \\
Female & $98(55)$ \\
Follow-up duration (months) & $12.6($ range 0-71.6) \\
Primary cancer & \\
Lung cancer & $58(33)$ \\
Breast cancer & $45(25)$ \\
Hepatocellular carcinoma & $13(7)$ \\
Renal cell carcinoma & $12(7)$ \\
Prostate cancer & $11(6)$ \\
Colorectal cancer & $10(6)$ \\
Thyroid cancer & $7(4)$ \\
Bladder/ureter cancer & $5(3)$ \\
Sarcoma & $5(3)$ \\
Others & $12(7)$ \\
Chemotherapy & \\
Preoperative & $42(24)$ \\
Postoperative & $31(17)$ \\
Both & $95(53)$ \\
No & $10(6)$ \\
Radiation therapy & \\
Independent & $32(18)$ \\
Noncurrent & $84(47)$ \\
Survival & $62(35)$ \\
Yes & \\
No & $159(89)$ \\
\hline
\end{tabular}

hypertension or both, and/or unexpected loss of consciousness occurring around the time of cementation, prosthesis insertion, reduction of the joint, or limb tourniquet deflation in a patient undergoing cemented bone surgery." BCIS was classified according to severity of symptoms: grade 1 was moderate hypoxia $(\mathrm{SpO} 2<94 \%)$ or hypotension (fall in SBP $>20 \%$ ); grade 2 was severe hypoxia ( $\mathrm{SPO} 2<88 \%$ ) or hypotension (fall in $\mathrm{SBP}>40 \%$ ) or unexpected loss of consciousness; and grade 3 was cardiovascular collapse requiring cardiopulmonary resuscitation (CPR). ${ }^{22,23}$ Pulmonary embolism caused by thrombus, fat, tumor emboli, or bone cement was investigated with chest computed tomography (CT). For the 23 patients who did not undergo postoperative chest CT, 16 were evaluated with positron emission tomography-computed tomography (PET-CT) and 7 with plain chest radiographs. The embolism was evaluated by a pulmonary imaging specialist (HL) and an orthopedic oncologist 
(JWP). For pulmonary embolism, clinical or radiological factors that might be related to its occurrence were collected, including primary cancer type, presence of lung metastasis, injected cement amount and number, local cement leakage during the procedure, pathologic fracture at the site for procedure, and injected route number.

\section{Surgical Technique}

The procedure was mainly performed under spinal anesthesia. The patient was placed in the lateral decubitus position with the surgical side up. The needle entry point was adjusted with a fluoroscopic guide near the point 10 $\mathrm{cm}$ posterior to the anterior superior iliac spine. The skin and bone were punctured by a 11-gauge needle (11-gauge, CAPO needle, Nexone Bio, Sung-nam, Korea). At least two or three different routes to the lesion were established, and one needle remained empty to allow decompression if possible (Fig. 2). Low-viscosity radiopaque bone cement (Exolent Spine, Elmdown, London, England) was quickly mixed and transferred to 1-ml syringes to reduce injection resistance. Injection was initiated 2-4 min after mixing. When the resistance to cement injection was high in the beginning, the cement was pushed in with a stylet, and it went well afterward. As much bone cement as possible was injected while avoiding leakage into the hip joint, sciatic notch, or veins under fluoroscopic monitoring. When significant cement leakage occurred at the initial stage, cement injection was performed via another route or was stopped and resumed when the cement viscosity increased. During the injection of PMMA, the anesthesiologist carefully monitored the change in blood pressure and pulse rate, given the known toxicity of bone cement. The cement began to harden after $8 \mathrm{~min}$, and the operator, therefore, aimed to complete the delivery of the injection within 10 min. The injection needles were removed before the cement hardened completely (Fig. 3).

\section{Statistical Analysis}

Continuous variables were compared using the independent-samples, and categorical variables were compared using Pearson's $\chi^{2}$ test. For continuous variables in which values were repeatedly measured, a paired $t$-test was used. Paired proportions were compared using McNemar test. The Bonferroni correction was applied to evaluate statistical significance in multiple comparisons. Clinical or radiological factors that were found to have a statistically significant association with the occurrence of BCIS and pulmonary cement embolism $(p<0.05)$ on univariate logistic regression analysis were included in a multivariate logistic regression analysis with backward selection using the likelihood ratio test to evaluate associations with this complication. Statistical analysis was performed using SPSS v. 21.0 software (IBM Inc., Armonk, New York).

\section{RESULTS}

\section{Surgical Outcome}

The mean regional pain scores (numerical rating scale; NRS) significantly decreased to 3.2 and 2.1 at 1 week and 1 month after procedure, respectively, compared with 6.1 before the procedure $(p<0.01$ for all pairs, using

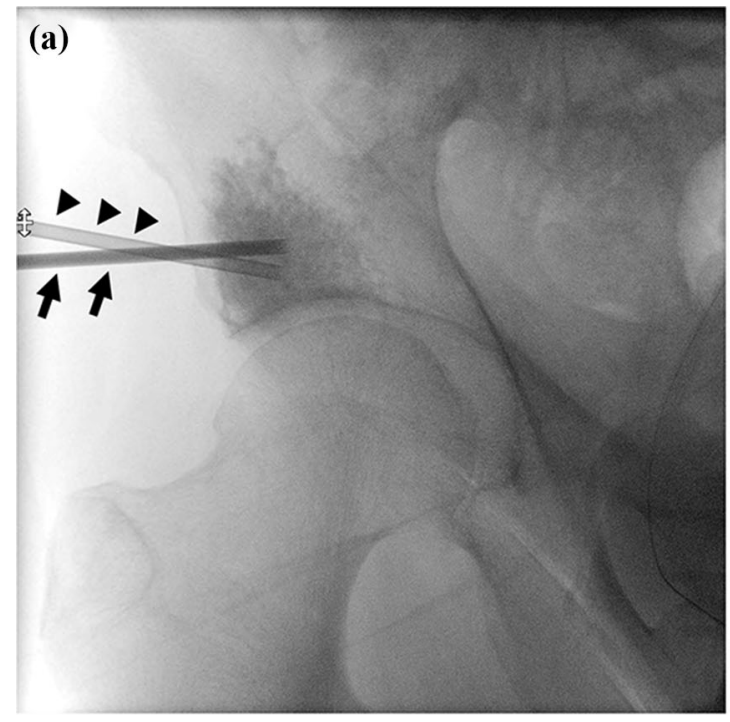

FIG. 2 The routes for percutaneous pelvic cementoplasty. a A fluoroscopic image and $\mathbf{b}$ a photograph showing that two routes were established for injection and decompression. The dark needle

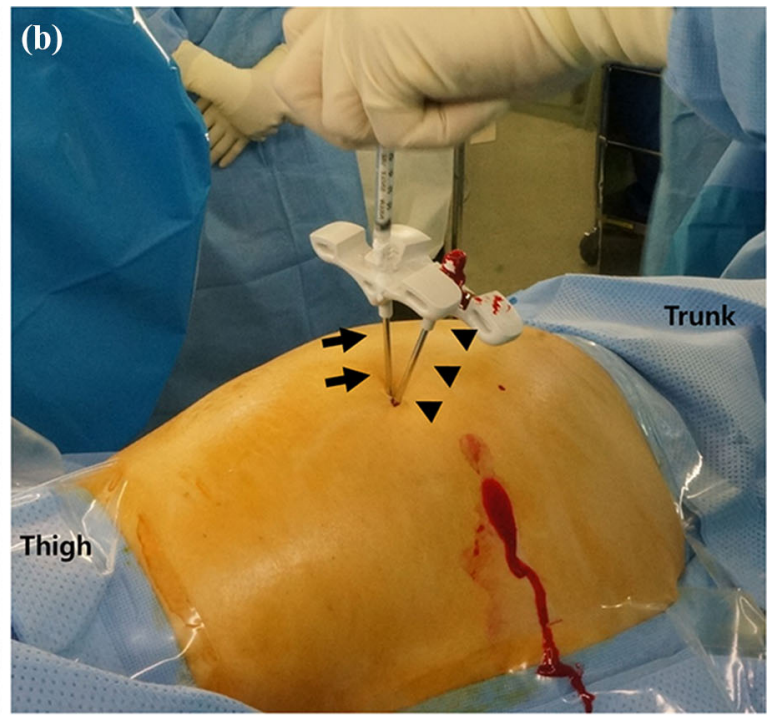

(arrows), which was filled with the bone cement, was used for injection, and the empty needle (arrowheads) was used for intraosseous decompression 

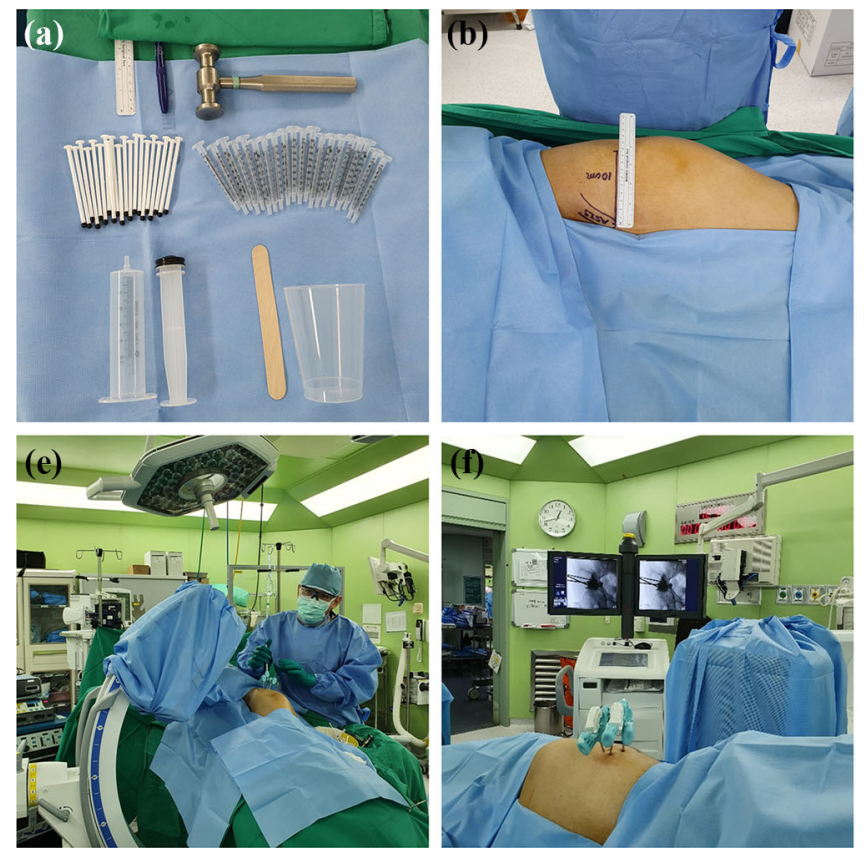

FIG. 3 Percutaneous pelvic cementoplasty. a Preparation for the procedure. b, $\mathbf{c}$ The three injection or decompression routes were established near the point $10 \mathrm{~cm}$ posterior to the anterior superior iliac spine. d A fluoroscopic image showing well-placed needles before

Bonferroni correction). The mean reduction in pain score was 2.9 at 1 week and 4.0 at 1 month postoperatively, which indicated a clinically important change $(>2)$ in acute pain. ${ }^{24}$ The percentage of patients with tolerable pain $(\mathrm{NRS} \leq 4)$ significantly increased to $70 \%$ and $88 \%$ at 1 week and at 1 month after the procedure, respectively ( $p<$ 0.01 for all pairs, using Bonferroni correction, Table 2).

One-month postoperative ambulatory function was classified as follows: normal or with a limping gait (42\%), independent gait with a crutch or cane (23\%), wheelchair ambulation (15\%), bed-ridden (12\%), or dead (8\%). There was significant increasing proportion for independent ambulatory status with/without a crutch or cane $(53 \%$ to $65 \%, p<0.01)$. However, the proportions of independent ambulation without crutch (49\% to $42 \%, p=0.37)$ and of bed-ridden status (9\% to $12 \%, p=0.13$ ) were not changed significantly. Notably, the patients in the group of the wheelchair ambulation preoperatively $(n=67)$ showed the greatest gait function improvement (improved 58\%, maintained $22 \%$, worsened $11 \%$, and uncheckable 9\%). Overall, gait functional status improved in $28 \%$ of patients, was maintained in $40 \%$, worsened in $24 \%$ despite the palliative procedure, and dead in $8 \%$ (Table 2). The patients who showed worsened gait function had brain metastases at the time of the procedure, and this was statistically significant $(p=0.03)$. However, there was no significant relationship with other clinical factors such as primary cancer type, spine metastasis, other wight-bearing
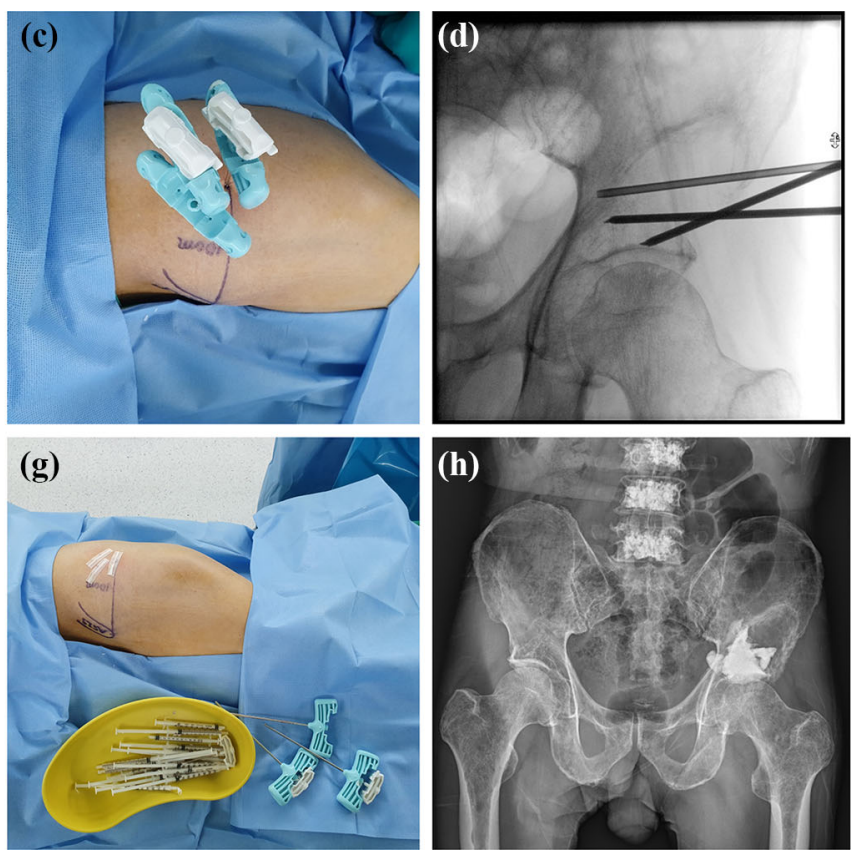

bone cement injection. e, f Bone cement injection was delivered under fluoroscopic guidance with $1 \mathrm{ml}$ syringes. $\mathrm{g}$ Status after the procedure. $\mathbf{h}$ Immediate postoperative plain radiograph showing the reinforced acetabular dome

bone metastasis, simultaneous surgery of other sites, and bone cement leakage during the procedure.

For the long-term survivors $(n=53)$ who were followed up for more than 12 months, there was no significant interval change in serial plain radiographs. Of these patients, regional pain aggravation (NRS $>3$ ) was observed in five patients (9\%). However, no patient underwent revisional surgery at the same location.

\section{Complications}

Regarding local complications, extraosseous bone cement leakage was often observed $(36 \%, 72 / 201$ of pelvises). Among them, 21 showed intraarticular leakage into the hip joint and 51 showed leakage into areas other than the hip joint. The main cause of the leakage was the pathological fracture of the pelvis, including impending fracture and displaced fracture $(p<0.01)$. However, extraosseous cement leakage was not related to postoperative pain or gait function.

For systemic complications, pulmonary embolism due to thrombus, fat, or injectable bone cement was investigated. There was no thromboembolism or fat embolism, but pulmonary cement embolism was detected in 19 patients (11\%). On univariate analysis, local venous cement leakage [odds ratio (OR) 4.21, $p<0.01$ ] and injection site number (OR 4.42, $p=0.01$ ) were significant predictive factors for pulmonary cement embolism. However, there 
TABLE 2 Surgical outcome

\begin{tabular}{ll}
\hline Characteristics & Value \\
\hline Pain (NRS, mean) & \\
Preoperative & 6.1 (range 0-10) \\
At 1 week postoperatively & 3.2 (range 0-9) \\
At 1 month postoperatively & 2.1 (range 0-9) \\
Tolerable pain (NRS $\leq 4)(n, \%)$ & \\
Preoperative & $47(26)$ \\
At 1 week postoperatively & $124(70)$ \\
At 1 month postoperatively & $134(88)$ \\
Gait function (n, \%) & \\
Preoperative & \\
Independent & $88(49)$ \\
Crutch or cane & $7(4)$ \\
Wheelchair & $67(38)$ \\
Bed-ridden & $16(9)$ \\
At 1 month postoperatively & \\
Independent & $74(42)$ \\
Crutch or cane & $41(23)$ \\
Wheelchair & $27(15)$ \\
Bed-ridden & $21(12)$ \\
Uncheckable & $15(8)$ \\
Gait function change $(n, \%)$ & \\
Improved & $49(28)$ \\
Stable & \\
Worsened & $43(24)$ \\
Uncheckable & $15(8)$ \\
\hline &
\end{tabular}

was no significant relationship between the pulmonary cement embolism and primary cancer type $(p=0.72)$ or hypervascularity of the primary cancer $(p=0.79)$. In addition, injection needle number (OR $0.48, p=0.16$ ) was the only factor that showed a tendency to protect against pulmonary cement embolism. On multivariate analysis, venous leakage (OR 3.83,p=0.01) and injection site number (OR 4.06, $p=0.02$ ) remained as significant independent predictive factors of pulmonary cement embolism (Table 3, Fig. 4).

For BCIS, there were 17 patients who met the criteria. Among 17 patients, 6 patients experienced hypoxia (4 of grade 1 and 2 of grade 2), 10 had hypotension ( 9 of grade 1 and 1 of grade 2), and a patient showed both (grade 1 hypoxia and grade 1 hypotension). For hypoxia, there were nine patients who showed hypoxia $\left(\mathrm{SpO}_{2}<94 \%\right)$ during the procedure but two patients who had had poor lung function before the procedure $\left(\mathrm{SpO}_{2} 84 \%\right.$ and $\left.87 \%\right)$ without sudden $\mathrm{SpO}_{2}$ drop during the procedure, excluded from BCIS. All seven patients with sudden hypoxia during the procedure recovered to their preoperative status without ventilator treatment; five recovered within $6 \mathrm{~h}$ and two required oxygen supply with nasal prong at $6 \mathrm{~h}$ after the procedure. There were 11 patients who experienced hypotension during the procedure, 10 who recovered to preoperative SBP in $6 \mathrm{~h}$, and 1 patient who showed $33 \%$ of reduction for SBP in $6 \mathrm{~h}$ postoperatively, but no patients required inotropics or intensive care unit. There were no patients with unexpected unconsciousness or cardiac arrest. Among all 17 patients with BCIS, there was no early death event after the procedure. Mean survival of the BCIS patients was 11.9 (range 0.5-77.6) months, 9.4 (range 0.5-77.6) months, and 23.9 (range 2.6-38.2) months for total, grade 1 , and grade 2 , respectively. Clinical and surgical factors were not significantly related to BCIS.

Nine patients died within 1 month after procedure. Of them, two showed cement pulmonary embolism on postoperative chest $\mathrm{CT}$, but no patient showed aggravation of dyspnea or desaturation in the immediate postoperative period compared with that before procedure. All nine patients died from disease progression, and there was no evidence of death from surgical complications. Six patients died during hospitalization, and two patients died from uncontrolled pneumonia with bronchial collapse due to lung lesions. Another patient was discharged after acute recovery but died due to pleural metastasis that progressed rapidly.

\section{DISCUSSION}

Pain reduction and functional outcomes after minimal invasive approach for the metastatic pelvic lesions in this paper were compatible with previous literature. Percutaneous cementoplasty for the acetabulum was introduced by Cotton et al. ${ }^{16}$ In the previous literature, the pain control effect had been consistently reported. ${ }^{4,14,16,25}$ As a minimally invasive procedure, there have been attempts to use radiofrequency ablation or cryotherapy alone, or in combination with the percutaneous cementoplasty. Recently, a minimally invasive method was used to combine metal fixation with screws in a minimally invasive manner. ${ }^{16,25}$ Theoretically, tumor ablation could suppress tumor regrowth. Ballooning to secure space for cement injection and adding metal fixation are expected to strengthen the treatment site. The common disadvantage of adding minimally invasive procedures such as radiofrequency ablation, cryotherapy, ballooning, and screw fixation was that the operation time was prolonged by tens of minutes to more than an hour and additional equipment was required for the procedures. There is no literature comparing the minimal invasive procedures or combination so far, but at least there seems to be no major difference in the evaluation of pain and gait function. ${ }^{4,14,16,25}$ In this study, there were no 
TABLE 3 Factors associated with pulmonary cement embolism

\begin{tabular}{|c|c|c|c|c|c|c|}
\hline \multirow[t]{2}{*}{ Factors } & \multicolumn{3}{|c|}{ Univariate } & \multicolumn{3}{|c|}{ Multivariate } \\
\hline & OR & $95 \% \mathrm{CI}$ & $p$-Value & OR & $95 \% \mathrm{CI}$ & $p$-Value \\
\hline Age & 0.98 & $0.94-1.02$ & 0.33 & & & \\
\hline Primary cancer & & & 0.72 & & & \\
\hline Lung cancer & 1.00 & 0.00 to inf. & & & & \\
\hline Breast cancer & 1.00 & 0.00 to inf. & & & & \\
\hline HCC & 1.00 & 0.00 to inf. & & & & \\
\hline $\mathrm{RCC}$ & 1.00 & 0.00 to inf. & & & & \\
\hline Prostate & 1.00 & 0.00 to inf. & & & & \\
\hline Others & 1.00 & & & & & \\
\hline Hypervascular tumor & & & 0.79 & & & \\
\hline HCC, RCC, thyroid & 0.84 & $0.23-3.08$ & & & & \\
\hline Others & 1.00 & & & & & \\
\hline Lung metastasis & & & 0.22 & & & \\
\hline Absent & 1.00 & & & & & \\
\hline Present & 1.84 & $0.70-4.88$ & & & & \\
\hline Total cement amount & 1.01 & $0.97-1.05$ & 0.65 & & & \\
\hline Venous leakage & & & $<0.01$ & & & 0.01 \\
\hline Absent & 1.00 & & & 1.00 & & \\
\hline Present & 4.21 & $1.57-11.32$ & & 3.83 & $1.39-10.55$ & \\
\hline Extraosseous leakage & & & 0.26 & & & \\
\hline Absent & 1.00 & & & & & \\
\hline Present & 1.74 & $0.67-4.52$ & & & & \\
\hline Pathologic fracture & & & 0.48 & & & \\
\hline Absent & 1.00 & & & & & \\
\hline Present & 0.48 & $0.18-1.29$ & & & & \\
\hline Injection needle number & & & 0.16 & & & \\
\hline 1 & 1.00 & & & & & \\
\hline 2 or more & 0.48 & $0.18-1.32$ & & & & \\
\hline Injection site number & & & 0.01 & & & 0.02 \\
\hline 1 & 1.00 & & & 1.00 & & \\
\hline 2 or more & 4.42 & $1.40-13.90$ & & 4.06 & $1.27-13.00$ & \\
\hline
\end{tabular}

additional procedures besides percutaneous cementoplasty, and we focused on minimizing the operation time. Nevertheless, there were no mechanical failures and few pain relapses $(9 \%)$ even in the long-term survivors (follow-up $>$ 1 year). Because activity demand in the advanced cancer patient was low, cementoplasty only seems to be sufficient to withstand it. Patients' general condition and life expectancy, preoperative gait function, activity demand, risk of an increase in surgery time, and expected benefits from adding procedures should be considered comprehensively to decide the optimal minimal invasive procedure for each patient.

In terms of radiotherapy timing, the patients were divided into three subgroups: independent, concurrent, and no radiotherapy. For all subgroups, pain score showed uniformly significant decrease 1 week and 1 month after the procedure (pre, 1-week, 1-month NRS score, 6.1, 3.1, 2.1 for independent; 6.1, 3.1, 2.1 for concurrent; 6.1, 3.0, 1.8 for no radiotherapy, and $p<0.01$ for all pairs, using Bonferroni correction). The amount of pain reduction appeared to be the greatest in the patients who did not receive RT, but this was not statistically significant. This may be due to the clinical policy that immediate postoperative RT was often omitted in patients whose reinforced pelvis was maintained relatively well with the procedure alone. Pain reduction appears to be more dependent on the procedure itself than on radiotherapy in this study.

Both complete and impending (a loss of pelvis cortex) pathologic fractures identified using MRI were the main causes of local bone cement leakage. This relationship between fracture and local cement leakage in this study is compatible with previous studies on vertebroplasty or kyphoplasty. ${ }^{26,27}$ In particular, a cortical defect at the acetabulum on the preoperative MRI was a strong 

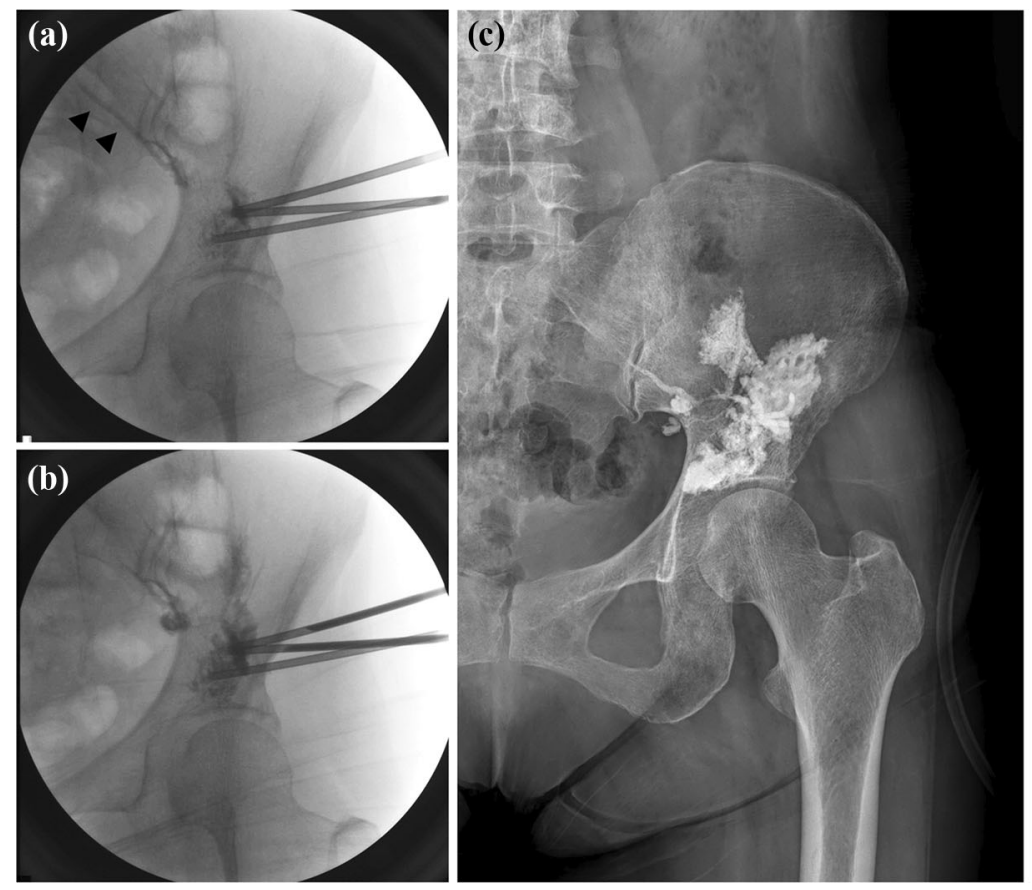
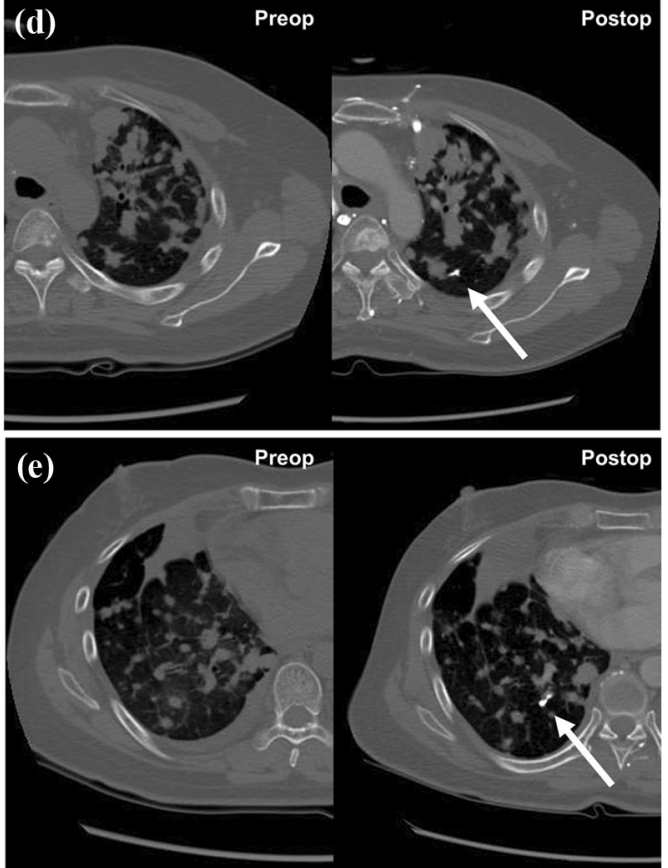

FIG. 4 Venous leakage and pulmonary cement embolism. a, b Serial fluoroscopic images showing venous leakage (arrowheads) that was washed out. c Postoperative plain radiographs revealing the reinforced acetabulum with venous leakage. d, e Preoperative (left)

predictive factor for intraarticular leakage of the hip $(p<$ $0.01)$. Among the 21 patients who showed intraarticular leakage, 17 had acetabular fractures or cortical defects at the acetabulum and 4 did not. Additional surgical techniques such as stopping and resuming cement injection or using a different injection route were required, although all 21 patients with intraarticular leakage were clinically asymptomatic, including hip joint pain or limitation of motion of the hip. The finding that intraarticular leakage had no impact on postoperative pain or symptoms was compatible with previous literature. ${ }^{4,14,25}$ Therefore, pathological fracture or cortical defect around the pelvic lesion required additional attention during percutaneous cementoplasty but was not a contraindication of the procedure.

In the present study, pulmonary embolism by thrombus or fat was not observed, but cement embolism was detected in $11 \%$ of patients. To date, there have been no previous studies on the incidence of pulmonary cement embolism related to percutaneous cementoplasty for the pelvis using a large group of patients. Several studies have reported the incidence of pulmonary cement embolism after vertebroplasty or kyphoplasty in the range of $2.1-26 \%{ }^{28,29}$ Like vertebral venous plexus, most external and internal iliac veins do not have a valvular structure, ${ }^{30}$ and this is likely and postoperative (right) pulmonary computed tomography images on the same level with the bone setting (level: $300 \mathrm{HU}$, width: $2000 \mathrm{HU}$ ) showing new pulmonary cement embolisms (white arrows)

why the incidence of pulmonary cement embolism after pelvic cementoplasty is similar to that after vertebroplasty or kyphoplasty.

The incidence of pulmonary cement embolism was not very low, but there were no patients who presented with dyspnea or desaturation. The number of cement emboli was minimal, and all the cement emboli were of the peripheral type. The effect of the presence of pulmonary cement emboli on patient overall health was unclear. However, it seemed there was a certain risk of pulmonary cement embolism regardless of the specific injection location or side, and the risk increased cumulatively when multiple sites were injected at the same time. Theoretically, having a decompression route reduces the difference between intraosseous pressure and atmospheric pressure. The incidence of pulmonary cement embolism was $17 \%(7 / 42)$ in patients undergoing single-route injection, while it was $9 \%$ $(12 / 136)$ in patients undergoing injection via more than two routes. However, there was no statistical significance $(p=$ 0.16). Unlike vertebroplasty or kyphoplasty, which use secure injection routes through vertebral pedicles, the pelvic bone is relatively wide, and it is possible to make multiple routes to the medullary space easily and safely. Therefore, in most procedures performed in this study, two or more routes were established, and bone cement was injected with intraosseous decompression. This may explain why the number of cement emboli was minimal 
and pulmonary cement embolism did not cause serious adverse effects in this cohort, despite having an incidence of $11 \%$.

The findings of this study should be interpreted in the context of some limitations. First, there was no control group in this study. The clinical indications for the procedure included pain and poor gait function, along with a poor general health condition that was contraindicative of major pelvic surgery. Due to ethical problems, a randomized control trial comparing the surgical procedure with a "wait and see" strategy was not possible. The authors performed the most appropriate treatment for each patient considering the patient's general condition, cancer stage, and life expectancy, so patients receiving other treatments in a similar clinical setting, that might have acted as a control group, were scarce. Second, comorbidities in the study population varied, and many factors remained uncontrolled. The presence and severity of brain/spinal cord metastasis, cardiac/pulmonary dysfunction, and numerous conditions related to chemotherapy were not uniformly distributed when pain and ambulatory status were evaluated. Third, the effect of intramedullary decompression was theoretically suggested without experimental support. Fourth, the single-institution design may limit its generalizability.

Percutaneous cement injection for the pelvis is a feasible and safe palliative surgical option for patients with advanced malignancy in terms of pain reduction and maintenance of ambulatory function under regional anesthesia. Although the incidence of pulmonary cement embolism as a complication was not low, it was not found to be clinically significant either.

ACKNOWLEDGMENTS This work was supported by the National Research Foundation of Korea (NRF) grant funded by the Korean government (MSIT) (No. 2020R1C1C1010776).

FUNDING This work was supported by the National Research Foundation of Korea (NRF) grant funded by the Korean government (MSIT) (No. 2020R1C1C1010776).

DISCLOSURE All authors declare that there are no conflicts of interest.

\section{REFERENCES}

1. Kiatisevi P, Sukunthanak B, Pakpianpairoj C, Liupolvanish P. Functional outcome and complications following reconstruction for Harrington class II and III periacetabular metastasis. World $J$ Surg Oncol. 2015;13:4.

2. Krishnan CK, Han I, Kim HS. Outcome after surgery for metastases to the pelvic bone: a single institutional experience. Clin Orthop Surg. 2017;9(1):116-25.
3. Marco RA, Sheth DS, Boland PJ, Wunder JS, Siegel JA, Healey $\mathrm{JH}$. Functional and oncological outcome of acetabular reconstruction for the treatment of metastatic disease. J Bone Joint Surg Am. 2000;82(5):642-51.

4. Muller DA, Capanna R. The surgical treatment of pelvic bone metastases. Adv Orthop. 2015. https://doi.org/10.1155/2015/ 525363.

5. Shinoda Y, Sawada R, Yoshikawa F, et al. Factors related to the quality of life in patients with bone metastases. Clin Exp Metastasis. 2019;36(5):441-8.

6. Basile A, Giuliano G, Scuderi V, et al. Cementoplasty in the management of painful extraspinal bone metastases: our experience. Radiol Med. 2008;113(7):1018-28.

7. Kim JH, Kang HG, Kim JR, Lin PP, Kim HS. Minimally invasive surgery of humeral metastasis using flexible nails and cement in high-risk patients with advanced cancer. Surg Oncol. 2011;20(1):e32-7.

8. Lane MD, Le HBQ, Lee $\mathrm{S}$, et al. Combination radiofrequency ablation and cementoplasty for palliative treatment of painful neoplastic bone metastasis: experience with 53 treated lesions in 36 patients. Skeletal Radiol. 2011;40(1):25-32.

9. Choi ES, Han I, Cho HS, Park IW, Park JW, Kim HS. Intramedullary nailing for pathological fractures of the proximal humerus. Clin Orthop Surg. 2016;8(4):458-64.

10. Kang HG, Roh YW, Kim HS. The treatment of metastasis to the femoral neck using percutaneous hollow perforated screws with cement augmentation. $J$ Bone Joint Surg $\mathrm{Br}$. 2009;91(8):1078-82.

11. Park JW, Kim YI, Kang HG, Kim JH, Kim HS. Preliminary results: use of multi-hole injection nails for intramedullary nailing with simultaneous bone cement injection in long-bone metastasis. Skeletal Radiol. 2019;48(2):219-25.

12. Kim YI, Kang HG, Kim SK, Kim JH, Kim HS. Clinical outcome prediction of percutaneous cementoplasty for metastatic bone tumor using (18)F-FDG PET-CT. Ann Nucl Med. 2013;27(10):916-23.

13. Li Z, Butala NB, Etheridge BS, Siegel HJ, Lemons JE, Eberhardt AW. A biomechanical study of periacetabular defects and cement filling. J Biomech Eng. 2007;129(2):129-36.

14. Maccauro G, Liuzza F, Scaramuzzo L, et al. Percutaneous acetabuloplasty for metastatic acetabular lesions. BMC Musculoskelet Disord. 2008;9:66.

15. Choi ES, Kim YI, Kang HG, Kim JH, Kim HS, Lin PP. Percutaneous cementoplasty for acetabulum in patients with bone metastasis. Acta Orthop Belg. 2017;83(3):480-487.

16. Cotten A, Deprez X, Migaud H, Chabanne B, Duquesnoy B, Chastanet P. Malignant acetabular osteolyses: percutaneous injection of acrylic bone cement. Radiology. 1995;197(1):307-10.

17. Durfee RA, Sabo SA, Letson GD, Binitie O, Cheong D. Percutaneous acetabuloplasty for metastatic lesions to the pelvis. Orthopedics. 2017;40(1):e170-5.

18. Gupta AC, Hirsch JA, Chaudhry ZA, et al. Evaluating the safety and effectiveness of percutaneous acetabuloplasty. J Neurointerv Surg. 2012;4(2):134-8.

19. Liu HF, Wu CG, Tian QH, Wang T, Yi F. Application of percutaneous osteoplasty in treating pelvic bone metastases: efficacy and safety. Cardiovasc Intervent Radiol. 2019;42(12):1738-44.

20. Georgy BA. Percutaneous cement augmentations of malignant lesions of the sacrum and pelvis. AJNR Am J Neuroradiol. 2009;30(7):1357-9.

21. Woo A, Lechner B, Fu T, Wong CS, Chiu N, Lam H, Pulenzas N, Soliman H, DeAngelis C, Chow E. Cut points for mild, moderate, and severe pain among cancer and non-cancer patients: a literature review. Ann Palliat Med. 2015;4(4):176-83. 
22. Donaldson AJ, Thomson HE, Harper NJ, Kenny NW. Bone cement implantation syndrome. $\mathrm{Br} J$ Anaesth. 2009; 102(1):12-22.

23. Hines, C. B. (2018). Understanding bone cement implantation syndrome. AANA J, 86(6).

24. Farrar JT, Berlin JA, Strom BL. Clinically important changes in acute pain outcome measures: a validation study. J Pain Symptom Manage. 2003;25(5):406-11.

25. Lee FY, Latich I, Toombs C, Mungur A, Conway D, Alder K, Ibe I, Lindskog D, Friedlaender G. Minimally invasive image-guided ablation, osteoplasty, reinforcement, and internal fixation (AORIF) for osteolytic lesions in the pelvis and periarticular regions of weight-bearing bones. J Vasc Interv Radiol. 2020;31(4):649-658.e1.

26. Tuan TA, Luong TV, Cuong PM, Long V, Huy HQ, Duc NM. Cement leakage in percutaneous vertebroplasty for multiple osteoporotic vertebral compression fractures: a prospective cohort study. Orthop Res Rev. 2020;12:105-11.
27. Xie W, Jin D, Ma H, et al. Cement leakage in percutaneous vertebral augmentation for osteoporotic vertebral compression fractures: analysis of risk factors. Clin Spine Surg. 2016;29:E171-6.

28. Krueger A, Bliemel C, Zettl R, Ruchholtz S. Management of pulmonary cement embolism after percutaneous vertebroplasty and kyphoplasty: a systematic review of the literature. Eur Spine J. 2009;18(9):1257-65.

29. Wang LJ, Yang HL, Shi YX, Jiang WM, Chen L. Pulmonary cement embolism associated with percutaneous vertebroplasty or kyphoplasty: a systematic review. Orthop Surg. 2012;4(3):182-9.

30. LePage PA, Villavicencio JL, Gomez ER, Sheridan MN, Rich NM. The valvular anatomy of the iliac venous system and its clinical implications. J Vasc Surg. 1991;14(5):678-83.

Publisher's Note Springer Nature remains neutral with regard to jurisdictional claims in published maps and institutional affiliations. 